\title{
Ideal guidance and procedures for donning and doffing personal protective equipment to be used by health workers during management of patients confirmed with COVID-19 or person under investigation
}

\author{
Saima Ahmad*, Haseeb Manzoor, Fatima Gul Chaudhary
}

Diagnostic and Interventional Neuroradiology Department, Lahore General Hospital, Lahore, Pakistan

Received: 06 May 2020

Revised: 26 July 2020

Accepted: 30 July 2020

\section{*Correspondence:}

Dr. Saima Ahmad,

E-mail: masterinfluencer@gmail.com

Copyright: () the author(s), publisher and licensee Medip Academy. This is an open-access article distributed under the terms of the Creative Commons Attribution Non-Commercial License, which permits unrestricted non-commercial use, distribution, and reproduction in any medium, provided the original work is properly cited.

\begin{abstract}
Highly infectious disease epidemics such as COVID-19 put healthcare workers at higher risk of infection than regular people as they come into more potential contact with contaminated respiratory droplets and so caution is warranted in the use of personal protective equipment (PPE) to lessen risk. It is not clear what kind of PPE best offers protection and the best way to remove PPE. PPE is used by Healthcare workers in the COVID-19 virus disease situation. Its use is an integral part of the strategy to combat COVID-19 and prevent its spread. Optimal PPE use is hard and thus healthcare workers may alter the delivery of care because of improper PPE use. Preventing contamination of PPE during donning and doffing therefore becomes crucial and requires improvisation. Donning and doffing of PPE should include adherence to protocols as well as COVID- 19 specific design of PPE and further research into the risks, benefits, and best practices of PPE use. Lastly, training of users must be stressed to minimize protocol deviations and in turn guarantee the best protection to HCWs. While ranked as the lowest in infection control hierarchy due to its effectiveness as compared to other measures and high cost, PPE is nonetheless extremely important and often overlooked aspect during early outbreak stages of a pandemic where neither drugs nor vaccines are available and access to them is limited. This paper sheds light on the donning and doffing protocols that should be used as well as discusses this often-overlooked aspect of the virus control measure.
\end{abstract}

Keywords: Health care workers, Donning, Doffing, Personal protective equipment, COVID-19

\section{INTRODUCTION}

It is an urban legend that the US surgeon general in the late 1960's Dr. William Stewart was known to say that time to close the book on infectious diseases and declare the war against pestilence won. ${ }^{1}$

13 December, 2019 saw a cluster of pneumonia cases of unknown etiology in Wuhan, China; by January 9, 2020 CDC China had reported a novel coronavirus in the SARS-CoV phylogenetic clade as the cause of the epidemic the disease is now called COVID-19 or novel corona virus.

Spread or transmission is through respiratory droplets, through inhalation or deposition on mucosal surfaces and contact with contaminated fomites and aerosol-generating procedures. It has also been isolated from respiratory, fecal, and blood specimens. ${ }^{2}$

Evidence shows that COVID-19 is a respiratory disease different from the Ebola virus disease which is 
transmitted through infected bodily fluids according to the last updated version of WHO. The personal protective equipment (PPE) required for COVID-19 is different from Ebola as coveralls are not needed. Evidence so far shows that COVID-19 is transmitted through droplets and not via airborne transmission. ${ }^{3}$

An important component in the control strategy to protect HCWs (health care workers), PPE is a vital component. Through preventing contamination of garments and hands, PPE aims to prevent infection in HCWs as well as to secondary patients. If we can understand how and where HCW contamination occurs it is important for improving PPE and infection control practices for both routines and specialized patient care. ${ }^{4}$ PPE selection is based on one of two criteria either route of disease transmission identification or through standard precautions..$^{5}$ As COVID-19 is a new virus and it is yet to be confirmed whether its transmission is airborne, we have to decide about PPE accordingly.

This outbreak has shed light on important gaps in PPE use and implementation strategies in HCWs in preventing disease transmission to patients although setting, equipment, and instructions for donning and doffing practices in COVID-19 is different yet insight from other scenarios can be applied here.

\section{GUIDELINES}

The following guidance on the types of PPE to be used and the processes for donning and doffing PPE is for all personnel who are treating patients hospitalized with COVID-19. Many HCW die in areas where there is an epidemic break out because of the high risks of infection and fatality rates. As per the statistics from the 2013-2015 West Africa EVD epidemics, there were 1049 cases of $\mathrm{HCW}$ infected with almost half of them dying. A decade ago healthcare workers lost their lives due to the SARS epidemic. ${ }^{6}$ This guideline reflects lessons learned from these past experiences and stresses the importance of training in donning and doffing of PPE.

All PPE must be used in the context of a comprehensive infection control program. To protect HCWs caring for COVID-19 patients, the facility should provide on-site management of and adherence to safety measures for PPE use. Administrative and environmental controls must be implemented with continuous safety checks.

\section{Environmental control for $\mathrm{HCW}$}

Proper administrative procedures and safe work practices should be carried out in relevant physical settings and include; identification, before time, the collection of lab specimens and the management of waste disposal should be done for critical patient care functions. Ensure training and evaluation of HCWs is mandatory before they go into the patient care area. PPE is selected based on a risk assessment and the needed medical clearance, maintenance, training, storage, reporting, etc are in place for workers who have potential exposure.

\section{Practices}

Donning and doffing procedures should be practiced by HCWs before they care for a COVID-19 Patient. Shift lengths tolerance and involved stresses must be gauged through practicing simulated patient care activities while wearing PPE. The use of a trained observer to ensure PPE correct use. Those unable to correctly follow PPE procedures should not be allowed to care for COVID-19 patients. PPE donning and doffing areas should be done in designated spaces to prevent cross-contamination. Frequent disinfection of gloved hands by using alcoholbased disinfectant. Use needleless I/V systems where possible.

\section{PRINCIPLES OF PPE}

The basic principles listed below should be followed by HCWs to ensure safety.

\section{Donning}

PPE must be donned in the correct order and not modified later inpatient care area. The activity should be carried out in the presence of a trained observer. During patient care, HCW should always be wearing PPE when the inpatient care area and in case of exposure to significant coughing and sneezing with droplet migration, the worker should be moved to doffing area immediately and remove the PPE. Gloved hands must be disinfected with ABHR after every contact with the patient. The HCW must be moved to the doffing area immediately to access exposure if a breach in PPE occurs (a needle prick, tear in outer gloves or a glove separating from the sleeve) during patient care. If a PPE breach (breach in outer glove, glove separates from the sleeve, a needle prick) happens during patient care the worker should be moved to doffing area.

\section{Doffing}

A designated area for protection, a trained observer and a doffing assistant are needed for the removal of PPE which is a very high-risk procedure. The outer gloves get grossly contaminated during patient care and double gloving is an easy way to avoid harm to the HCW.

\section{Training on correct usage of PPE}

The following elements are essential for PPE training. How to safely don, adjust, use and doff the specific PPE? How to safely conduct clinical care limitations? What to do in case of detection of a breach and how to dispose appropriately after use?

\section{Designating areas for PPE donning and doffing}

Hospitals should make sure that there is a clear separation between clean and infected areas. Establish a one-way 
flow of care moving from clean areas to patient care area and to PPE removal area. Donning area is a place where clean PPE is stored and where HCWs don PPE before entering the patient care area. No contaminated equipment should be present here.

PPE doffing area is a designated area where HCW can stand to doff and get rid of PPE when leaving. In this area, disinfection supplies for PPE and hand hygiene should be available as well as a space to get rid of PPE including ease access to cleansed and disinfected seats for HCW to remove boot covers. A container to collect reusable PAPR components should also be present in this area.

The facility should select and standardize the PPE to be used by all HCWs who are in direct contact with COVID19 patients.

\section{VIRAL TRANSMISSION}

Direct and indirect contact of the mucosae with infectious respiratory droplets and fomites is the biggest route of person to person spread of COVID-19. Nosocomial transmission of COVID-19 is facilitated by nebulizers, intubation, suction, bronchoscopy, and cardiopulmonary resuscitation. Even though airborne transmission of COVID-19 is considered uncommon, this pandemic is known to have transmitted through commercial flights. The pattern of involvement is atypical given the short duration of exposure of a few hours and the large involvement of patients sitting at distant rows. Airborne transmission is the only likely explanation here and this lesson was learned from the SARS-CoV outbreak.

Use oxygen via low flow nasal cannula rather than high flow masks to reduce airborne transmission risk. Negative pressure isolated rooms with strict airborne precautions should be used for non-invasive mechanical ventilation such as positive airway pressure use. ${ }^{4}$

CDC recommends all $\mathrm{HCWs}$ to wear respiratory protection such as fit-tested N95 or N99 or a PAPR while entering the patient care area.

Comfort and safety are both crucial for HCWs wearing PPE when caring for COVID-19 patients. The standardization of attire under the PPE such as surgical scrubs or disposable garments and dedicated washable footwear can make the donning and doffing processer easier. Footwear should be washable and have a closed back.

\section{RECOMMENDED PPE WHEN CARING FOR A DIAGNOSED AND SUSPECTED PATIENT OF COVID-19.}

\section{Impermeable garment}

The gown should be available in all sizes so that people can cover their forearms and arms without having a gap between the gloves and sleeves. Gowns must have thumb hooks and gloves can also be taped with the gown, but scissors should not be used to remove tape as it heightens the risk of contamination.

A study on SARS-CoV found that it stays longer on cotton gowns in comparison to disposable ones and thus absorbent materials like cotton should be preferred over non-absorptive materials for PPE clothing in routine patient care. ${ }^{4}$

Appropriate protective clothing makes it possible to form a barrier that eliminates or reduces contact and droplet exposure and thus prevents the transfer of microorganisms between patient and $\mathrm{HCW}$. Isolation gowns are used by HCW are either disposable or reusable. Isolation gowns are typically constructed of non-woven material alone or in combination with materials that offer increased protection from liquid penetration, such as plastic films. Non-woven fiber bonding technologies (thermal, chemical or mechanical) provide integrity rather than interlocking geometrics associated with knitted material. Disposable or reusable isolation gowns are used by HCW. The isolation gown is typically constructed of non-woven material or in combination with materials that allow increased protection from liquid penetration like plastic film. Nonwoven fiber bonding technologies provide integrity instead of interlocking geometrics of knitted materials.

The reusable isolation gowns are normally $100 \%$ polyester, $100 \%$ cotton, or a blend of the two. They are laundered after each use and impermeable gowns are the gold standard as they don't allow vapors through. ${ }^{7}$

\section{RESPIRATORY PROTECTION}

Either a powered air-purifying respirator (PAPR) or disposable National Institute for Occupational Safety and Health (NIOSH) certified N95 respirators must be used when aerosol-generating procedures need to be performed in an emergency. PAPR with a head shroud and face fully covered can cause accidentally self-contamination for example while adjusting eyeglasses. The disposal of N95 face respirators are easier and can be doffed safely. Tight fitted respirators on the other hand cannot be used by people with facial hair that interferes with facial seal.

PAPR, a hooded respirator, hood piece, or a helmet with a full-face shield. A single-use disposable hood that extends to the shoulders must cover any reusable helmet and completely covers the neck along with compatibility with the relevant PAPR.

\section{N95 respirator}

N95 respirator or higher in combination with single use disposable, surgical hood extending to the shoulder and single-use full-face shield. If N95 is used HCW should be 
observed to ensure they don't touch their face under the shield.

Single-use examination gloves with long cuffs, two pairs of gloves should be used so that the soiled outer gloves can be removed and replaced during care.

Disposable boot covers that extend to the mid-calf in combination with ankle-high shoe covers should be worn over the boot covers and may help in the doffing process. Single-use shoe covers are only acceptable when used in combination with integrated socks.

Disposable apron that covers the torso to the level of the mid-calf can be worn over the gown if the patient is known to have diarrhea in COVID-19. An apron should be selected based on having an unprotected zipper and neck strap that can easily be broken.

\section{Recommended sequences for donning PPE donning PPE, PAPR option}

The procedure assumes the facility has elected to use PAPRs. Training and compliance are facilitated through established protocols and a trained observer should verify compliance of said protocols.

Removal of personal clothing and items; users should have no personal items like watches, pens, papers or jewelry and should change into dedicated washable footwear and surgical scrubs in a suitable clean area. Long hair and eyeglasses should be secured.

Inspect PPE before donning, observer should review the sequence of donning with the healthcare worker before the donning process and read all the steps out loud as they happen. PPE should be visually inspected to ensure all supplies are available and there is no breach in PPE.

Put on boot covers, if a gown without integrated socks is used, the upper band of the boot cover will be worn under the pants leg. This step is not necessary if using a gown with integrated socks. Put on inner gloves, put on the first pair of gloves. Put on gown, the gown should be big enough to allow unrestricted movement and the cuffs of the inner gloves should be tucked under the sleeve.

If a PAPR with a blower unit and self-contained filter is integrated within the helmet, then a belt and the battery unit should be placed before donning to keep them under the gown. If PAPR has an external blower then it should be placed outside the gown. Put on outer gloves, a second pair of long cuff gloves should be used, and the cuffs must be pulled over the gown sleeves. Put on the respirator, a PAPR with helmet, face-shield or headpiece should be put on. In case of a PAPR with a self-contained filter and blower within the helmet a single hood extending to the shoulder and neck should be worn. In the case of a PAPR with an external belt-mounted blower and attached disposable headpiece, a single-use hood extending to the shoulders and neck should be used. Put on the outer apron (optional), single use apron can be used. Verify the HCW should be able to extend his arms be mobile and able to bend while completely covered for proper patient care and a mirror should be there in the donning room for them to see.

\section{Donning PPE, N95 respirator option}

This process assumes the facility is using the N95 respirator. All steps are similar but, instead of PAPR, put on the N95 Respirator and check the seal.

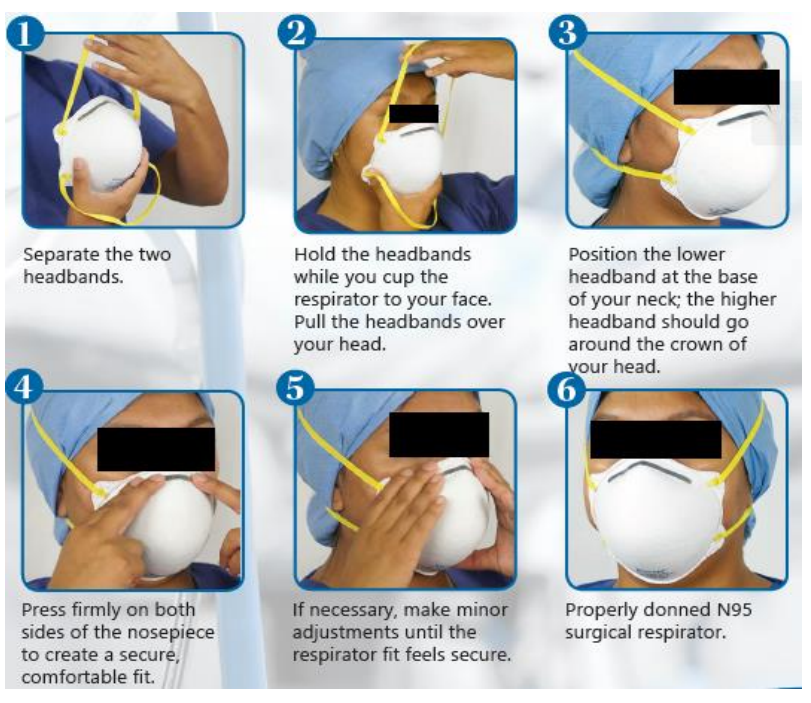

Figure 1: Donning and doffing of respirator N95.

\section{Doffing PPE, PAPR option}

Designated PPE removal area should be used for doffing and all PPE waste should be placed in a leak-proof infectious waste container.

A trained observer, reading out loud each step should visually confirm the doffing process. The healthcare worker should doff PPE while the observer coaches and reminds the healthcare worker to avoid reflexive actions like touching the face.

The PPE should be accessed for visible contamination, tears, or cuts before starting to remove. If there is contamination it should be cleaned and disinfected using a disinfectant, then and there. Disinfect outer-gloved hands with ABHR and allow them to dry. Remove apron (if used), by untying neck strap and roll it away from you. Discard apron taking care to avoid contaminating gloves or other surfaces. After removal inspect the PPE ensemble. In case of contamination, disinfect the contaminated area.

Disinfect outer gloved hands with ABHR (alcohol based hand rub). Remove and discard outer gloves ensuring that inner gloves are not contaminated in the process. Inspect the inner gloves for visible contamination. If an inner 
glove is contaminated disinfect it with ABHR, remove it, and perform hand hygiene and put on a new pair of gloves. If there is no contamination, then disinfect the gloves with ABHR.

Remove respirator with external belt-mounted blower, the observer (buddy) should help the healthcare worker in removing the headpiece. All reusable PAPR should be placed in an area designated for the collection of PAPR components for disinfection. Disinfect inner gloves with ABHR.

Remove gown and discard. The healthcare worker should untie fasteners, have the doffing assistant, or "buddy" unfasten the gown, avoiding contact of scrubs or disposable garments with the outer surface of gown during removal. Disinfect inner gloves with ABHR. Remove boot covers by sitting on a clean new chair pull off boot covers, taking care not to contaminate pants legs. Disinfect washable shoes, use disinfectant to wipe down every external surface of the washable shoes. Disinfect inner gloves with ABHR. Remove respirator (if not already removed), If a PAPR with a self-contained blower in the helmet is used, remove all remaining components here. If N95 respirator is used Remove N95 Respirator: Remove the respirator by tilting the head slightly forward, grasping first the bottom tie or elastic strap, then the top tie or elastic strap, and remove without touching the front of the respirator. Discard N95 respirator. Disinfect innergloved hands with ABHR. Remove and discard gloves, taking care not to contaminate bare hands. Perform hand hygiene with ABHR. The trained observer and healthcare worker should perform a final inspection of the worker for contamination of disposable garments. If contaminated, the garments should be carefully removed, and the wearer should shower immediately. The trained observer should immediately inform the infection prevention or occupational safety and health coordinator. Healthcare workers can leave the PPE removal area, proceeding directly to the showering area where his safeties are removed.

Protocol evaluatio/medical assessment should be done on a regular basis by the occupational safety and health coordinator to review patient care activities performed and healthcare workers' level of fatigue.

\section{CRISIS MANAGEMENT DURING COVID-19 PANDEMIC}

Desperate times call for desperate measures. We are already getting reports from hospitals on a shortage of equipment needed to care for critically ill patients, including ventilators and personal protective equipment (PPE) for medical staff. Flattening the curve of this pandemic may take months.

N95 filtering facepiece respirators and surgical masks are commonly used for protection from fine airborne particles known to be associated with various respiratory diseases.
One of the smallest known bioaerosol agents, viral particles, has a diameter ranging from 20 to $300 \mathrm{~nm}$. A certified N95 respirator can stop them penetrating through and thus the efficiency of the respirator as calculated must be at least $95 \%$. Surgical masks let a protection against aerosolized infectious agents in the size range of 10 to 80 $\mathrm{nm}$ and it should be noted that these masks are designed to protect the environment from the wearer while N95 protects the wearer from the environment. ${ }^{8}$ The coronavirus is roughly $70-220 \mathrm{~nm}$ in size and therefore the N95 provides protection from it. ${ }^{9}$

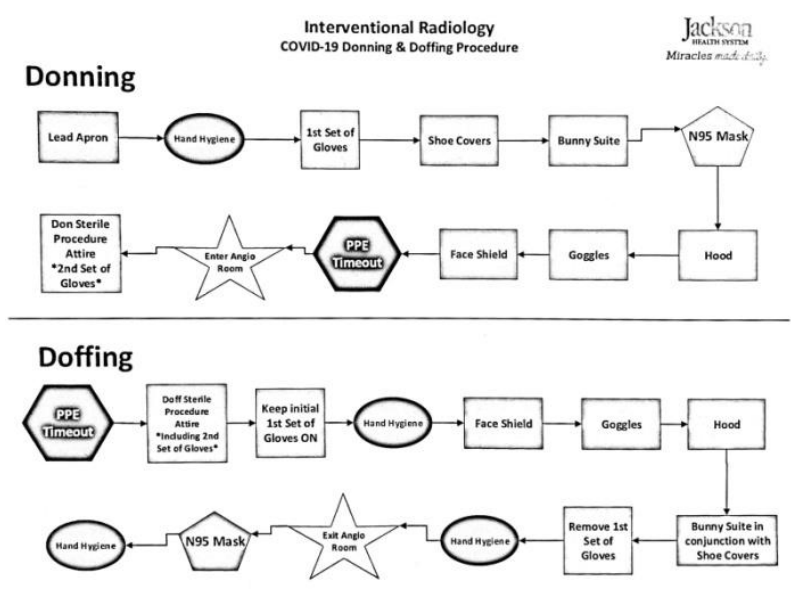

Figure 2: Donning and doffing PPE during interventional radiological procedures.

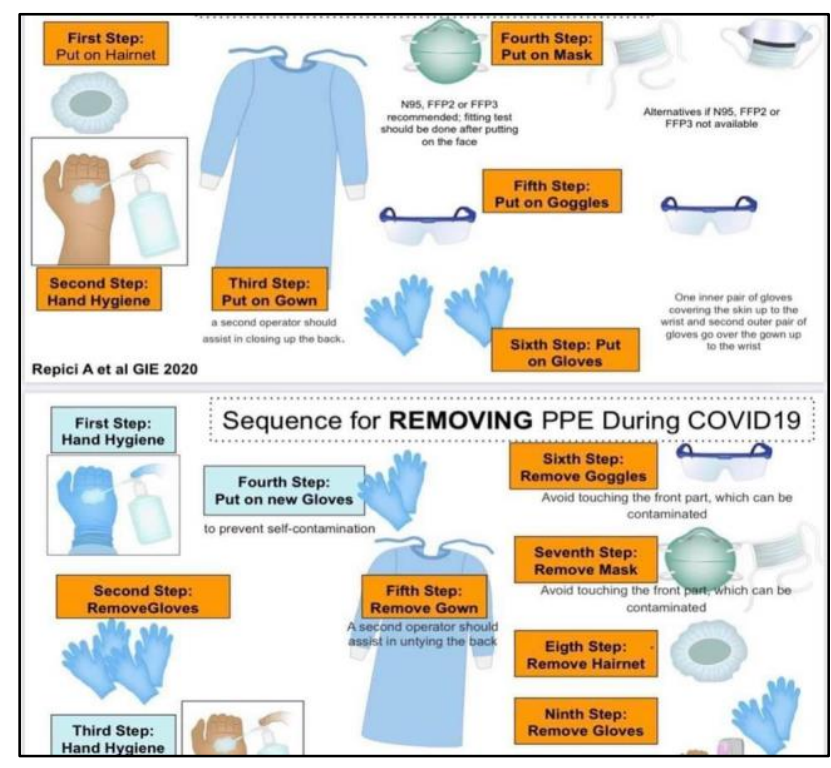

Figure 3: Steps for donning and doffing PPE during COVID-19. ${ }^{16}$

The centre for disease control (CDC) and prevention during the crises recommended the use of N95 masks only during aerosol-generating procedures, but that puts health care workers at risk as using less protective surgical masks around patients with confirmed or suspected COVID-19 infection. Furthermore, the CDC guideline includes reusing masks and respirators intended 
for one-time-only use and should the stocks be depleted using scarves and bandanas. No evidence to support these recommendations exists. The solution for PPE is that the private-sector companies play their part in making more PPE. U.S factories must increase production while the FDA reduces its standards and eases approval of these N95's using the National Institute for Occupational Safety and Health guidelines instead. ${ }^{10}$

Other strategies to improve N95 supply includes isolating patients, creating physical barriers like plastic or glass windows at reception areas, curtains between patients, etc. and most importantly maintaining a ventilation system to provide air movement from a clean to the contaminated flow direction. ${ }^{11}$

HCW's also having other options of impermeable protective clothing in the marketplace. Isolation gowns should be kept in mind when finding the most appropriate clothing and employees should know the limitations and available options. HCWs can use disposable patient isolation gowns that routine patients use when caring for patients with suspected or confirmed COVID-19. In shortages, surgical gowns should be a priority for healthcare activities for COVID-19 patients. $^{12}$

Reusable gowns are mostly polyester or polyester-cotton fabrics. The gowns can be safely laundered according to routine procedures and reused. Their outer surfaces should not however be touched and HCWs can use expired gowns beyond manufacturer-designated shelf life. Cloth isolation gowns can be untied and retied and can be reused without laundering in-between. Multiple COVID19 patients can be examined and treated in a single disposable gown. ${ }^{12}$ In the case of the absence of gowns consider using lab-coats, reusable washable laboratory coats, and reusable patients gowns. ${ }^{12}$

When no additional eye shields are available eye shields can be reprocessed during crisis management. While wearing gloves, they can be wiped on the inside followed by the outside of the face shield or goggles using a clean cloth saturated with neutral detergent solution or cleaner wipe. Using a wipe or clean cloth saturated with hospital disinfectant solution goggles and the outside of the face shield should be carefully wiped. The outside of the face shield or goggles can also be wiped with clean water or alcohol to remove residue and fully dried with clean towels. $^{13}$

\section{DISCUSSION}

As evidenced by a study of PPE doffed by volunteers who were contaminated with bacteriophage MS2, a nonenveloped, non-pathogenic RNA virus that fluoresces under UV light, performing a healthcare task. Then asked to remove the PPE according to the CDC protocols. The sites of contamination found were front shoulder of gown, back shoulder of gown, the palm of the dominant hand, upper right front of goggles, and the right side of the N95 respirator. ${ }^{14}$

Another study conducted on 12 cases of COVID-19 who had endotracheal intubation at the Guangzhou eighth hospital from January to February 2020. The data from procedures was collected and analyzed. Nine workers involved in the procedure had daily monitoring of temperature for 14 days and had a COVID-19 test done. All 12 patients were incubated with a bronchoscope. Morphine, midazolam and propofol were used for sedation and to prevent patients from coughing or agitation. 9 healthcare workers were protected with PPE with positive pressure protective hood.

Oropharyngeal swab virus nucleic acid was found to be negative in all healthcare workers and there was no fever or respiratory symptoms. The PPE with positive pressure protective hood is mandatory while performing bronchoscope guided endotracheal intubation in patients with COVID-19. The study shows that the use of PPE among healthcare workers for their own protection. ${ }^{15}$

While HCWs take precautions against airborne, droplet, and contact precautions in a negative pressure room, no additional personal protective equipment like powered air-purifying respirators (PAPRs) need to be used. The logic behind this being that prior research shows that unfamiliar or increasingly complex PPE increases the risk of self- contamination. As illustrated by the Canadian report, while the novelty of PPE "maximalism" should be avoided in favour of proven strategies that $\mathrm{HCW}$ practice and conduct with a high degree of fidelity without selfcontamination. The WHO and others have issued helpful guidance on this rational use of PPE for COVID-19, aiming to optimize $\mathrm{HCW}$ safety while mitigating disruption in the global supply chain of PPE. Furthermore, preventing rushes on the public market through measured risk communication with the public can safeguard needed supplies. Finally, strategies to reduce less urgent use of PPE and find situations where different types of protection can be used where elements of PPE can be reused. ${ }^{16}$

Another study showed that N95 respirators, disinfection, and hand washing reduce the infectious risk of 2019$\mathrm{nCoV}$ in healthcare professionals. It was also seen that high male doctor proportions seemed to have a higher risk of infection. The results emphasize the need for strict occupational protection measures to fight COVID-19. ${ }^{16}$

\section{CONCLUSION}

It is important to note that hand hygiene is critical. A barrier to improving this has been the belief that gloves make it unnecessary. It contradicts various studies that show organisms can spread from glove to hand after glove removal. Even if double gloving protocols are in place for PPE, it is not a substitute for proper hand hygiene. The medical community has shown great effort 
in understanding COVID-19 in a short amount of time as shown by the many publications, but the transmutability of the virus is still not clear. Coronavirus is known to undergo genetic mutation evidenced by SARS-CoV and COVID-19. The availability of large CoV like viruses in bats, along with a culture of eating exotic mammals in China is a time bomb that may lead to re-emergence of other Corona Viruses. Since this virus is novel and the whole world is affected by the pandemic, there are limited resources for PPE. Hence, this article provides safe ways to use already available resources and how to safely recycle and reuse them.

\section{ACKNOWLEDGEMENTS}

Authors are thankful to MT 2020 and Jackson memorial hospital, Miami, Florida for providing us with flow chart for donning and doffing PPE in interventional radiological procedures.

Funding: No funding sources

Conflict of interest: None declared

Ethical approval: Not required

\section{REFERENCES}

1. Weber DJ, Rutala, WA, Fischer WA, Kanamori H. and Sickbert-Bennett EE. Emerging infectious diseases: Focus on infection control issues for novel coronaviruses (Severe Acute Respiratory Syndrome$\mathrm{CoV}$ and Middle East Respiratory Syndrome-CoV), hemorrhagic fever viruses (Lassa and Ebola), and highly pathogenic avian influenza viruses, A (H5N1) and A (H7N9). American journal of infection control. 2016;44(5):e91-e100.

2. Cheng VC, Lau SK, Woo PC, Yuen KY. Severe acute respiratory syndrome coronavirus as an agent of emerging and reemerging infection. Clinical microbiology reviews. 2007;20(4):660-94.

3. Chughtai AA, Khan W. Use of personal protective equipment to protect against respiratory infections in Pakistan: A systematic review. Journal of infection and public health; 2002.

4. Prevention II. Control Recommendations for Patients with Confirmed Coronavirus Disease 2019 (COVID19) or Persons Under Investigation for COVID-19 in Healthcare Settings. Update: February; 2012: 21.

5. Casanova L, Alfano-Sobsey E, Rutala WA, Weber DJ, Sobsey M. Virus transfer from personal protective equipment to healthcare employees' skin and clothing. Emerging infectious diseases. 2008;14(8):1291.
6. Kilinc FS. A review of isolation gowns in healthcare: fabric and gown properties. Journal of engineered fibers and fabrics. 2015;10(3):155892501501000313.

7. Bałazy A, Toivola M, Adhikari A, Sivasubramani SK, Reponen T, Grinshpun SA. Do N95 respirators provide $95 \%$ protection level against airborne viruses, and how adequate are surgical masks?. American journal of infection control. 2006;34(2):51-7.

8. Ranney ML, Griffeth V, Jha AK. Critical supply shortages - the need for ventilators and personal protective equipment during the Covid-19 pandemic. New England Journal of Medicine. 2020;382(18):e41.

9. Mor N. Resources for primary care providers to meet patients needs during the COVID-19 epidemic; 2020.

10. Patel A, Bell M, De Perio M. CDC 2019 novel coronavirus response: Strategies for ensuring healthcare systems preparedness and optimizing N95 supplies; 2020.

11. Mahajan A, Hirsch JA. Novel coronavirus: what neuroradiologists as citizens of the world need to know; 2020.

12. Chughtai AA, Khan W. Use of personal protective equipment to protect against respiratory infections in Pakistan: A systematic review. Journal of infection and public health; 2020.

13. Cai SJ, Wu LL, Chen DF, Li YX, Liu YJ, Fan YQ, Du SH, Huang H, Liu N, Cheng LL, Deng XL. Analysis of bronchoscope-guided tracheal intubation in 12 cases with COVID-19 under the personal protective equipment with positive pressure protective hood. Zhonghua jie he he hu xi za zhi= Zhonghua jiehe he huxi zazhi= Chinese journal of tuberculosis and respiratory diseases; 2020.

14. Maxwell DN, Perl TM, Cutrell JB. "The Art of War" in the Era of Coronavirus Disease 2019 (COVID-19). Clinical infectious diseases: an official publication of the Infectious Diseases Society of America; 2020.

15. Wang $X$, Pan Z, Cheng Z. Association between 2019-nCoV transmission and N95 respirator use. medRxiv; 2020.

16. Repici A. GIE; 2020.

Cite this article as: Ahmad S, Manzoor H,

Chaudhary FG. Ideal guidance and procedures for donning and doffing personal protective equipment to be used by health workers during management of patients confirmed with COVID-19 or person under investigation. Int J Community Med Public Health 2020;7:4164-70. 